have been at least partially acclimatized to the more standard conditions of life in an aquarium.

Chapter one is entitled "T'owards a Valid Sampling Technique" and illustrates the problems encountered by research workers in obtaining animals in a sufficiently unstressed condition, from the sea or an aquarium, to detcrmine what might be regarded as a normal range of concentration. This difficulty is apparent in almost all of the subsequent chapters when the author goes on to discuss the quantitative and qualitative changes in the chemical composition that occur during the life cycle, the effect of changes in the external environment and the effects of voluntary or involuntary starvation. Next, sorne differences between species are discussed and finally there are two appendices. The first appendix occupies 4 pages and consists of a simple, useful but somewhat uncritical index to references where individual chemical compounds have been detected in a wide variety of fishes. The second, of 16 pages, details the familial relationships of fish genera and gives the common and the systematic name as well as some qualitative indication of the chemical compounds that have been identified in the animal.

The aim of the book has been to relate a chemical and an analytical approach to the general biology of fishes. The selection of material for discussion has obviously been influenced to some extent by the author's own extensive research work and his own personal viewpoint. This is all to the good because the text is clearly and often entertainingly written in a highly individual style, but it should be noted that the features of metabolism that distinguish fish from other animals and the nature of fish enzymes have not been considered in detail.

To sum up, this monograph is an extensive reference work on the chemical composition of fishes up to 1968. It will be a valuable source of information to research workers and students who have interests in marine biology.

$$
\text { P. T. Grant }
$$

\section{THE ANIMAL WORLD}

\section{Animal Senses}

By Robert Burton. Pp. $183+18$ plates. Charles: Newton Abbot, March 1970.) 65s.

(David and

There has always been a fascination in considering what the world must look, smell and sound like to animals. Is their world similar to ours, or is it a caricature of the detailed sensations which we feel ? With the pace of electrophysiological investigations on sense organ function and the quantity of information coming from experiments on perception, there is almost a continuing need for reviews of this subject couched in terms which the layman understands. The author of this book has undoubtedly set out with this aim in mind, but in execution has come sadly wide of the mark. There are too many misleading statements and inaccuracies for the book to be recommended. One way in which the author errs is in the misapplication of terms which have quite specific meanings; thus, the explanation of kinesis on p. 19 is quite misleading, the performance of a worm in a T-maze is not a demonstration of a conditioned reflex, and a catalyst does not "supply energy".

In line with modern ideas the author describes experiments on sense organs but too often leaves out important details; for example, one electrode is insufficient to detect impulses in a nerve; where is the other one? With the anatomy and function of sense organs, mistakes again appear. Thus the hearing organs of grasshoppers (if we take the popular English usage of the word) are not in the legs, and the vibrations striking the antenna of a male mosquito are not transmitted down it but the whole flagellum is set into motion and it is the tuning of this flagellum which makes it so responsive to the wing note of the female. The illustrations are often over-simplified sketches of the true complexity of the sense organs; glaring examples are the figures of the hearing organ of the grasshopper (which also shows the receptors in the wrong position) and of the facial pit of a viper.

One last strange feature of the book is the selection of entries in the bibliography; most of these are original papers which must be difficult for most of the potential readers to obtain. There are a number of short books which have been published within the past three years which would have been far more helpful for anybody looking for more information. J. D. CARThY

\section{DEFINING ECOLOGICAL CONCEPTS}

\section{Diversity and Stability in Ecological Systems}

Report of Symposium held on May 26-28, 1969. (Brookhaven Symposia in Biology, No. 22.) Pp. vii +264 . (Clearinghouse for Federal Scientific and Technical Information, NBS, US Department of Commerce: Springfield, Virginia.) $\$ 3 \cdot 0$.

ONE of the pressing problems facing theoretical ecologists is the construction of a precisely defined framework on which to hang their data. Ecology is still dogged by vague, quasi-philosophical terms which lend themselves to a multitude of individual interpretations; two of such terms are diversity and stability. These concepts, together with their relationship to one another, form the theme of a symposium held at Brookhaven, New York, in May 1969. This volume is a valuable collection of nineteen papers from that symposium, given by a number of leading ecologists, and it serves to emphasize the need for unified models of ecological systems which will allow precise, mathematical formulation of our current embryonic concepts of ccosystem function.

Most of the authors interpret diversity in terms of the MacArthur model and take stability to mean the ability of a system to withstand and absorb major environmental perturbations. Contention arises in the definition of a major perturbation, and Lewontin helps to elucidate this by constructing a theoretical model in which there are several possible "basins of attraction" into which a system can settle; thus when perturbation becomes sufficiently violent the system may move to a new basin and achieve a new equilibrium. Margalef goes further and proposes a function which expresses stability in terms of biomass and attrition of that biomass.

Other papers, for example, those by Miller, Sanders and Slobodkin, are concerned with the origins of diversity, and all conclude that environmental stress leads to lowered diversity, whereas predictable environments (for example, the sea bed) allow a highly diverse, biologically accommodated biota to develop. Wilson provides experimental demonstration of this principle in his study of arthropod invasion of defaunated mangrove islands.

The time factor in the definition of stability is a familiar problem and Goulden shows long term increases in diversity by a study of fossil Cladocera in lake sediments, a subject further elaborated by Deevey.

A number of field situations are analysed in detail, for example, Watt's work on the behaviour of populations of insects and of epidemic diseases. Ho demonstrates an interesting, if enigmatic, correlation between upsets in population stability and voleanic eruptions. Cantlon. concentrates on the effects of technology on natural populations of organisms, which provides a link with several papers dealing with the importance of stability and its accompanying (causative ?) diversity to the survival of man on this planet. Some profitable thoughts emerge from these papers, though attempts to elevate ecology to a political system ("biocracy"-Caldwell) or even a religion ("not peace--but ecology"-Hardin) must be viewed with caution.

The volume is sensibly priced, poorly arranged and well worth reading.
Peter D. Moore 